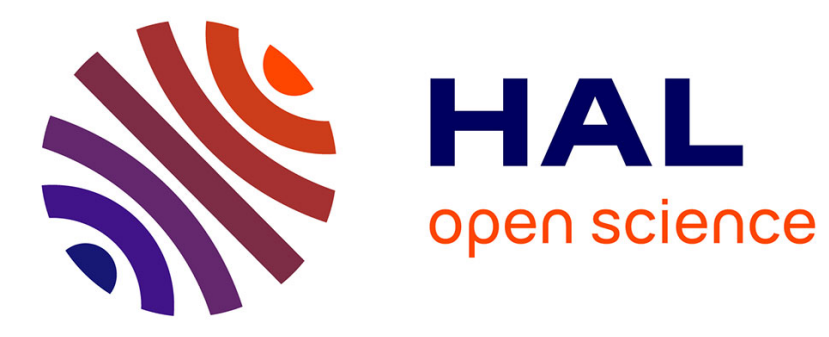

\title{
Une variante à la Coxeter du groupe de Steinberg
}

Christian Kassel, Christophe Reutenauer

\section{To cite this version:}

Christian Kassel, Christophe Reutenauer. Une variante à la Coxeter du groupe de Steinberg. KTheory, 1998, 14, pp.305-318. hal-00124660

\section{HAL Id: hal-00124660 \\ https://hal.science/hal-00124660}

Submitted on 15 Jan 2007

HAL is a multi-disciplinary open access archive for the deposit and dissemination of scientific research documents, whether they are published or not. The documents may come from teaching and research institutions in France or abroad, or from public or private research centers.
L'archive ouverte pluridisciplinaire HAL, est destinée au dépôt et à la diffusion de documents scientifiques de niveau recherche, publiés ou non, émanant des établissements d'enseignement et de recherche français ou étrangers, des laboratoires publics ou privés. 


\title{
Une variante à la Coxeter du groupe de Steinberg
}

\author{
Christian Kassel ${ }^{1}$ et Christophe ReutenaueR ${ }^{2}$ \\ 1 Institut de Recherche Mathématique Avancée, Université Louis Pasteur - C.N.R.S., 7 rue René Descartes, \\ 67084 Strasbourg Cedex, France \\ 2 Mathématiques, Université du Québec à Montréal, Montréal, CP 8888, succ. Centre Ville, Canada H3C $3 P 8$
}

ABSTRACT. Let $G_{n}(\Lambda)$ be the semi-direct product of the symmetric group $\mathfrak{S}_{n}$ by the Steinberg group $S t_{n}(\Lambda)$ of a ring $\Lambda$. We first prove that $G_{n}(\Lambda)$ has a Coxeter-type presentation. The canonical morphism $S t_{n}(\Lambda) \rightarrow G L_{n}(\Lambda)$ extends to a group homomorphism $\Phi: G_{n}(\Lambda) \rightarrow G L_{n}(\Lambda)$. We next determine the kernel of $\Phi$ for $n=\infty$. We also give an expression for the generator of the algebraic K-group $K_{2}(\mathbf{Z})$ of the integers in terms of permutation matrices.

Mathematics Subject Classification (1991): 19C09, 19C99, 20B30, 20F55

KEY WorDS: Steinberg group, $K_{2}$, infinite symmetric group, central extension of a group

RÉSUMÉ. Soit $G_{n}(\Lambda)$ le produit semi-direct du groupe symétrique $\mathfrak{S}_{n}$ par le groupe de Steinberg $S t_{n}(\Lambda)$ d'un anneau $\Lambda$. Nous établissons au théorème 1 que $G_{n}(\Lambda)$ a une présentation similaire à celle d'un groupe de Coxeter. L'application canonique $S t_{n}(\Lambda) \rightarrow G L_{n}(\Lambda)$ s'étend en un homomorphisme de groupes $\Phi: G_{n}(\Lambda) \rightarrow G L_{n}(\Lambda)$. Nous en déterminons le noyau dans le cas stable $n=\infty$ (voir Théorèmes 2 et 3). Au passage nous donnons une expression pour le générateur du groupe $K_{2}(\mathbf{Z})$ de K-théorie algébrique de l'anneau $\mathbf{Z}$ des nombres entiers relatifs à l'aide de matrices de permutation (voir Proposition 2).

Mots-ClÉs : Groupe de Steinberg, K-théorie algébrique, groupe symétrique, extension centrale de groupes 


\section{Introduction}

Le but de cet article est de montrer comment l'on peut passer des relations de Steinberg à une version paramétrée des relations de Coxeter formant la présentation standard des groupes symétriques. On y passe du groupe de Steinberg à un groupe un peu plus gros ayant une présentation à la Coxeter, et du groupe $K_{2}$ de K-théorie algébrique à un groupe qui joue un rôle équivalent pour le groupe à la Coxeter.

Considérons les matrices carrées $P_{i}(\lambda)(i=1, \ldots, n-1)$ de taille $n$, obtenues à partir de la matrice identité en insérant à l'intersection des lignes et colonnes $i$ et $i+1$ le bloc

$$
\left(\begin{array}{ll}
\lambda & 1 \\
1 & 0
\end{array}\right)
$$

dont le coefficient $\lambda$ est un élément d'un anneau $\Lambda$. Le point de départ de notre travail est l'observation que les matrices $P_{i}(\lambda)$ satisfont entre elles les trois relations de Coxeter "avec paramètres" suivantes :

$$
\begin{aligned}
P_{i}(\lambda) P_{i}(0) P_{i}(\mu) & =P_{i}(\lambda+\mu), \\
P_{i}(\lambda) P_{j}(\mu) & =P_{j}(\mu) P_{i}(\lambda) \quad \text { si }|i-j| \geq 2, \\
P_{i}(\lambda) P_{i+1}(\mu) P_{i}(\nu) & =P_{i+1}(\nu) P_{i}(\mu+\lambda \nu) P_{i+1}(\lambda) .
\end{aligned}
$$

Soit $G_{n}(\Lambda)$ le groupe engendré par des éléments $y_{i}^{\lambda}(i=1, \ldots, n-1, \lambda \in \Lambda)$ soumis aux trois relations précédentes. Le théorème 1 énonce que pour $n \geq 3$ le groupe ainsi défini est isomorphe au produit semi-direct du groupe symétrique $\mathfrak{S}_{n}$ par le groupe de Steinberg $S t_{n}(\Lambda)$ de l'anneau $\Lambda$.

Soit $\Phi$ l'homomorphisme de groupes de $G_{n}(\Lambda)$ vers le groupe $G L_{n}(\Lambda)$ des matrices inversibles donné par $\Phi\left(y_{i}^{\lambda}\right)=P_{i}(\lambda)$. Son image est le sous-groupe engendré par les matrices élémentaires et les matrices de permutation.

Nous déterminons le noyau $N(\Lambda)$ de l'homomorphisme $\Phi$ dans le cas stable, c'est-àdire après passage à la limite inductive sur $n$, et pour les anneaux dans lesquels la matrice d'une transposition n'est pas le produit de matrices élémentaires. La clé du calcul de $N(\Lambda)$ se trouve dans la suite exacte de groupes

$$
0 \rightarrow K_{2}(\Lambda) \rightarrow N(\Lambda) \rightarrow \mathfrak{A}_{\infty} \rightarrow 1
$$

où $\mathfrak{A}_{\infty}$ est le groupe alterné infini et $K_{2}(\Lambda)$ est le groupe de $K$-théorie algébrique de l'anneau $\Lambda$ tel qu'il a été défini par Milnor. Nous montrons que cette extension de groupes est centrale (corollaire 2) et que, si $\Lambda$ est l'anneau $\mathbf{Z}$ des entiers relatifs, le noyau $N(\mathbf{Z})$ est isomorphe à l'extension centrale universelle $\widetilde{\mathfrak{A}}_{\infty}$ de $\mathfrak{A}_{\infty}$ (théorème 2 ).

La démonstration du théorème 2 est une application de la théorie des extensions centrales de groupes. Elle fait apparaître quatre extensions centrales :

$$
\begin{gathered}
0 \rightarrow C \rightarrow \widetilde{\mathfrak{A}}_{\infty} \rightarrow \mathfrak{A}_{\infty} \rightarrow 1, \quad 0 \rightarrow K_{2}(\mathbf{Z}) \rightarrow N(\mathbf{Z}) \rightarrow \mathfrak{A}_{\infty} \rightarrow 1, \\
0 \rightarrow K_{2}(\mathbf{Z}) \rightarrow S t(\mathbf{Z}) \rightarrow E(\mathbf{Z}) \rightarrow 1 \quad \text { et } \quad 0 \rightarrow \mathbf{Z} / 2 \rightarrow S^{3} \times S^{3} \rightarrow S O(4) \rightarrow 1 .
\end{gathered}
$$


Ces extensions centrales non scindées ont en commun que leur noyau est isomorphe à $\mathbf{Z} / 2$ et que le générateur de celui-ci s'exprime, dans les quatre cas, simplement à l'aide des permutations $(12)(34)$ et $(13)(24) \in \mathfrak{A}_{4}$ ou des matrices de permutation correspondantes. En particulier, on obtient ainsi une nouvelle expression du générateur de $K_{2}(\mathbf{Z})$ (proposition 2).

Au théorème 3 nous déterminons $N(\Lambda)$ dans le cas général. Le calcul se ramène à celui de $N(\mathbf{Z})$.

Le paragraphe 1 est consacré à l'énoncé du théorème 1 et le paragraphe 2 à sa démonstration. Au paragraphe 3 nous considérons la limite inductive $G(\Lambda)$ des groupes $G_{n}(\Lambda)$ et nous énonçons nos résultats concernant le noyau $N(\Lambda)$ de l'homomorphisme $\Phi$. Enfin, le paragraphe 4 contient la démonstration du théorème 2 qui détermine $N(\mathbf{Z})$ ainsi que des calculs dans les quatre extensions centrales mentionnées plus haut.

\section{Le groupe $G_{n}(\Lambda)$}

Nous considérons le groupe de Steinberg $S t_{n}(\Lambda)$ avec les notations de [3], chap. $5 ; \Lambda$ est un anneau et $n \geq 3$. Ce groupe est engendré par les éléments $x_{i j}^{\lambda}, 1 \leq i, j \leq n, i \neq j$, $\lambda \in \Lambda$, sujets aux relations de Steinberg:

$$
\begin{array}{rlrl}
x_{i j}^{\lambda} x_{i j}^{\mu} & =x_{i j}^{\lambda+\mu}, & \\
x_{i j}^{\lambda} x_{k l}^{\mu} & =x_{k l}^{\mu} x_{i j}^{\lambda} & & \text { si } i \neq l \text { et } j \neq k, \\
x_{i j}^{\lambda} x_{j k}^{\mu} & =x_{j k}^{\mu} x_{i j}^{\lambda} x_{i k}^{\lambda \mu} & & \text { si } i \neq k .
\end{array}
$$

Le but premier de cet article est de montrer que ces relations peuvent être remplacées par des relations à la Coxeter au prix d'un léger agrandissement du groupe.

Le groupe symétrique $\mathfrak{S}_{n}$ opère $\operatorname{sur} S t_{n}(\Lambda)$ par ${ }^{\sigma} x_{i j}^{\lambda}=x_{\sigma(i) \sigma(j)}^{\lambda}$ pour $\sigma \in \mathfrak{S}_{n}$. Soit $G_{n}(\Lambda)$ le produit semi-direct de $\mathfrak{S}_{n} \operatorname{par} S t_{n}(\Lambda)$ correspondant à cette action. Un élément typique de $G_{n}(\Lambda)$ s'écrit sous la forme unique $w \sigma$ avec $w \in S t_{n}(\Lambda)$ et $\sigma \in \mathfrak{S}_{n}$, et le produit de deux tels éléments est donné par $w \sigma w^{\prime} \sigma^{\prime}=w^{\sigma} w^{\prime} \sigma \sigma^{\prime}$. De manière équivalente, on a $\sigma x_{i j}^{\lambda} \sigma^{-1}=x_{\sigma(i) \sigma(j)}^{\lambda}$ dans $G_{n}(\Lambda)$. Le groupe $S t_{n}(\Lambda)$ est un sous-groupe normal de $G_{n}(\Lambda)$ et le quotient $G_{n}(\Lambda) / S t_{n}(\Lambda)$ est isomorphe à $\mathfrak{S}_{n}$.

Définissons des éléments $y_{i}^{\lambda}$ de $G_{n}(\Lambda)$ par $y_{i}^{\lambda}=x_{i, i+1}^{\lambda} s_{i}$, où $s_{i}$ est la transposition $(i, i+1)$ de $\mathfrak{S}_{n}$ et $1 \leq i<n$.

THÉORÈme 1.- Le groupe $G_{n}(\Lambda)$ est engendré par les éléments $y_{i}^{\lambda}, i=1, \ldots, n-1, \lambda \in \Lambda$ et admet comme présentation

$$
\begin{aligned}
y_{i}^{\lambda} y_{i}^{0} y_{i}^{\mu} & =y_{i}^{\lambda+\mu}, \\
y_{i}^{\lambda} y_{j}^{\mu} & =y_{j}^{\mu} y_{i}^{\lambda} \quad \text { si }|i-j| \geq 2, \\
y_{i}^{\lambda} y_{i+1}^{\mu} y_{i}^{\nu} & =y_{i+1}^{\nu} y_{i}^{\mu+\lambda \nu} y_{i+1}^{\lambda} .
\end{aligned}
$$

Le groupe de Steinberg $S t_{n}(\Lambda)$ a été défini à partir des relations universelles satisfaites par les matrices élémentaires $e_{i j}^{\lambda}(1 \leq i \neq j \leq n$ et $\lambda \in \Lambda)$. Rappelons que $e_{i j}^{\lambda}$ est la matrice carrée $n \times n$ dont les coefficients sont nuls à l'exception du coefficient $(i, j)$ égal à $\lambda$ et des 
coefficients diagonaux égaux à 1 . Soit $\varphi: S t_{n}(\Lambda) \rightarrow G L_{n}(\Lambda)$ l'homomorphisme de groupes déterminé par $\varphi\left(x_{i j}^{\lambda}\right)=e_{i j}^{\lambda}$ pour tout $1 \leq i \neq j \leq n$ et $\lambda \in \Lambda$.

Il en va de même pour le groupe $G_{n}(\Lambda) \hat{\mathrm{E}}$ : les relations (1) du théorème 1 sont satisfaites par la famille de matrices $P_{i}(\lambda)(1 \leq i<n$ et $\lambda \in \Lambda)$ définies comme suit. Posons

$$
P(\lambda)=\left(\begin{array}{ll}
\lambda & 1 \\
1 & 0
\end{array}\right)=\left(\begin{array}{cc}
1 & \lambda \\
0 & 1
\end{array}\right)\left(\begin{array}{ll}
0 & 1 \\
1 & 0
\end{array}\right)
$$

pour $\lambda \in \Lambda$. Les matrices $P(\lambda)$ ont été introduites par Cohn (voir [1], Chap. 2) pour étendre l'algorithme d'Euclide à certains anneaux non commutatifs et pour donner une présentation du groupe $G L_{2}(\Lambda)$ d'une algèbre associative libre. Elles servent aussi au calcul des réduites des fractions continues usuelles.

La matrice $P_{i}(\lambda) \in G L_{n}(\Lambda)$ est définie comme la somme diagonale des matrices $I_{i-1}$, $P(\lambda)$ et $I_{n-i-1}$ où $I_{j}$ est la matrice identité d'ordre $j$. En d'autres termes, on a

$$
P_{i}(\lambda)=e_{i, i+1}^{\lambda} s_{i}
$$

où nous avons utilisé la même notation pour la transposition $s_{i}$ et pour la matrice de permutation correspondante.

Lemme 1.- Pour tout $\lambda, \mu, \nu \in \Lambda$ et tout $i, j=1, \ldots, n-1$ on a les relations

$$
\begin{aligned}
P_{i}(\lambda) P_{i}(0) P_{i}(\mu) & =P_{i}(\lambda+\mu), \\
P_{i}(\lambda) P_{j}(\mu) & =P_{j}(\mu) P_{i}(\lambda) \quad \text { si }|i-j| \geq 2, \\
P_{i}(\lambda) P_{i+1}(\mu) P_{i}(\nu) & =P_{i+1}(\nu) P_{i}(\mu+\lambda \nu) P_{i+1}(\lambda) .
\end{aligned}
$$

DÉMonstration. - La première relation a été observée par Cohn. La seconde est évidente. La troisième découle des identités

$$
\left(\begin{array}{lll}
\lambda & 1 & 0 \\
1 & 0 & 0 \\
0 & 0 & 1
\end{array}\right)\left(\begin{array}{lll}
1 & 0 & 0 \\
0 & \mu & 1 \\
0 & 1 & 0
\end{array}\right)\left(\begin{array}{lll}
\nu & 1 & 0 \\
1 & 0 & 0 \\
0 & 0 & 1
\end{array}\right)=\left(\begin{array}{ccc}
\mu+\lambda \nu & \lambda & 1 \\
\nu & 1 & 0 \\
1 & 0 & 0
\end{array}\right)
$$

et

$$
\left(\begin{array}{lll}
1 & 0 & 0 \\
0 & \nu & 1 \\
0 & 1 & 0
\end{array}\right)\left(\begin{array}{lll}
\xi & 1 & 0 \\
1 & 0 & 0 \\
0 & 0 & 1
\end{array}\right)\left(\begin{array}{ccc}
1 & 0 & 0 \\
0 & \lambda & 1 \\
0 & 1 & 0
\end{array}\right)=\left(\begin{array}{ccc}
\xi & \lambda & 1 \\
\nu & 1 & 0 \\
1 & 0 & 0
\end{array}\right)
$$

Il résulte du lemme qu'il existe un homomorphisme de groupes $\Phi: G_{n}(\Lambda) \rightarrow G L_{n}(\Lambda)$ défini par $\Phi\left(y_{i, i+1}^{\lambda}\right)=P_{i}(\lambda)$ pour tout $i$ et tout $\lambda \in \Lambda$. D'après la relation (2), la restriction de $\Phi$ au sous-groupe $S t_{n}(\Lambda)$ est l'homomorphisme $\varphi$ défini plus haut ; de plus, $\Phi$ envoie l'élément $s_{i}$ de $G_{n}(\Lambda)$ sur la matrice de permutation correspondante. 


\section{Démonstration du théorème 1}

Soit $H$ le groupe défini par la présentation du théorème 1 . Il s'agit de montrer que $H$ est isomorphe à $G_{n}(\Lambda)$.

Commençons par construire un homomorphisme de groupes $\psi: H \rightarrow G_{n}(\Lambda)$. Posons $y_{i j}^{\lambda}=x_{i, i+1}^{\lambda} s_{i} \in G_{n}(\Lambda)$ et montrons que les éléments $y_{i j}^{\lambda}$ vérifient les relations (1). Il en résultera que $\psi\left(y_{i j}^{\lambda}\right)=x_{i, i+1}^{\lambda} s_{i}$ définit bien un homomorphisme de groupes $\psi: H \rightarrow G_{n}(\Lambda)$.

(1.1) En utilisant les relations de Steinberg (S) nous avons

$$
\begin{aligned}
y_{i}^{\lambda} y_{i}^{0} y_{i}^{\mu} & =x_{i, i+1}^{\lambda} s_{i} \underbrace{x_{i, i+1}^{0}} s_{i} x_{i, i+1}^{\mu} s_{i} \\
& =x_{i, i+1}^{\lambda} \underbrace{s_{i} s_{i}} x_{i, i+1}^{\mu} s_{i} \\
& =\underbrace{x_{i, i+1}^{\lambda} x_{i, i+1}^{\mu}} s_{i} \\
& =x_{i, i+1}^{\lambda+\mu} s_{i} \\
& =y_{i}^{\lambda+\mu} .
\end{aligned}
$$

(1.2) Soit $i+1<j$. D'après les relations (S), la définition de l'action de $\mathfrak{S}_{n}$ sur $S t_{n}(\Lambda)$ et la commutation de $s_{i}$ et $s_{j}$, nous avons

$$
\begin{aligned}
y_{i}^{\lambda} y_{j}^{\mu} & =x_{i, i+1}^{\lambda} \underbrace{s_{i} x_{j, j+1}^{\mu}} s_{j} \\
& =\underbrace{x_{i, i+1}^{\lambda} x_{j, j+1}^{\mu}} \underbrace{s_{i} s_{j}} \\
& =x_{j, j+1}^{\mu} \underbrace{x_{i, i+1}^{\lambda} s_{j}} s_{i} \\
& =x_{j, j+1}^{\mu} s_{j} x_{i, i+1}^{\lambda} s_{i} \\
& =y_{j}^{\mu} y_{i}^{\lambda} .
\end{aligned}
$$

(1.3) Si la permutation $\sigma$ satisfait $\sigma(i)=j$ et $\sigma(i+1)=j+1$, alors $\sigma y_{i}^{\lambda} \sigma^{-1}=y_{j}^{\lambda}$. Par suite il suffit de vérifier la dernière des relations (1) pour $i=1$. Nous utilisons (S) à 
chaque étape. On a

$$
\begin{aligned}
& y_{1}^{\lambda} y_{2}^{\mu} y_{1}^{\nu}=x_{12}^{\lambda} s_{1} x_{23}^{\mu} \underbrace{s_{2} x_{12}^{\nu}} s_{1} \\
& =x_{12}^{\lambda} s_{1} \underbrace{x_{23}^{\mu} x_{13}^{\nu}} s_{2} s_{1} \\
& =x_{12}^{\lambda} \underbrace{s_{1} x_{13}^{\nu}} x_{23}^{\mu} s_{2} s_{1} \\
& =\underbrace{x_{12}^{\lambda} x_{23}^{\nu}} \underbrace{s_{1} x_{23}^{\mu}} s_{2} s_{1} \\
& =x_{23}^{\nu} x_{12}^{\lambda} \underbrace{x_{13}^{\lambda \nu} x_{13}^{\mu}} \underbrace{s_{1} s_{2} s_{1}} \\
& =x_{23}^{\nu} x_{12}^{\lambda} \underbrace{x_{13}^{\mu+\lambda \nu} s_{2}} s_{1} s_{2} \\
& =x_{23}^{\nu} \underbrace{x_{12}^{\lambda} s_{2}} x_{12}^{\mu+\lambda \nu} s_{1} s_{2} \\
& =x_{23}^{\nu} s_{2} \underbrace{x_{13}^{\lambda} x_{12}^{\mu+\lambda \nu}} s_{1} s_{2} \\
& =x_{23}^{\nu} s_{2} x_{12}^{\mu+\lambda \nu} \underbrace{x_{13}^{\lambda} s_{1}} s_{2} \\
& =\underbrace{x_{23}^{\nu} s_{2}} \underbrace{x_{12}^{\mu+\lambda \nu} s_{1}} \underbrace{x_{23}^{\lambda} s_{2}} \\
& =y_{2}^{\nu} y_{1}^{\mu+\lambda \nu} y_{2}^{\lambda} \text {. }
\end{aligned}
$$

Il s'agit maintenant de construire un homomorphisme de groupes $\chi: G_{n}(\Lambda) \rightarrow H$. Puisque $G_{n}(\Lambda)$ est le produit semi-direct de $\mathfrak{S}_{n}$ par $S t_{n}(\Lambda)$, il a la présentation suivante : les générateurs sont $x_{i j}^{\lambda}(1 \leq i \neq j \leq n$ et $\lambda \in \Lambda)$ et $s_{i}(1 \leq i<n)$; les relations sont celles de Steinberg $(\mathrm{S})$ pour les générateurs $x_{i j}^{\lambda}$, celles de Coxeter

$$
\begin{aligned}
s_{i}^{2} & =1, \\
s_{i} s_{j} & =s_{j} s_{i} \quad \text { si }|i-j| \geq 2, \\
s_{i} s_{i+1} s_{i} & =s_{i+1} s_{i} s_{i+1},
\end{aligned}
$$

pour les générateurs $s_{i}$, et les relations "mixtes"

$$
\sigma x_{i j}^{\lambda} \sigma^{-1}=x_{\sigma(i) \sigma(j)}^{\lambda}
$$

pour tout $\sigma \in \mathfrak{S}_{n}$, tout $\lambda \in \Lambda$ et tout $1 \leq i \neq j \leq n$. Ici l'on a identifié $\mathfrak{S}_{n}$ avec le groupe engendré par $s_{1}, \ldots, s_{n-1}$ et les relations $(\mathrm{C})$.

(C) Posons $\lambda=\mu=0$ dans les relations (1). Il en résulte que les éléments $s_{i}=y_{i}^{0}$ de $H$ vérifient les relations de Coxeter $(\mathrm{C})$.

(CS) Pour $\sigma \in \mathfrak{S}_{n}$ avec $\sigma(k)=i$ et $\sigma(k+1)=j$, posons (au prix d'un léger abus de notation) $x_{i j}^{\lambda}=\sigma y_{k}^{\lambda} s_{k} \sigma^{-1}$. Il faut vérifier que cet élément de $H$ est bien défini, i.e. que si $\sigma$ fixe $k$ et $k+1$, alors $\sigma y_{k}^{\lambda} s_{k} \sigma^{-1}=y_{k}^{\lambda} s_{k}$; mais dans ce cas, $\sigma$ commute à $y_{k}^{\lambda}$ et à $s_{k}$ (car $\sigma$ est un produit de $\left.s_{1}, \ldots, s_{k-2}, s_{k+2}, \ldots, s_{n-1}\right)$, d'où le résultat.

Vérifions maintenant que si $\alpha$ est une permutation, alors $\alpha x_{i j}^{\lambda} \alpha^{-1}=x_{\alpha(i) \alpha(j)}^{\lambda}$. On a

$$
\alpha x_{i j}^{\lambda} \alpha^{-1}=\alpha \sigma y_{k}^{\lambda} s_{k} \sigma^{-1} \alpha^{-1}=(\alpha \sigma) y_{k}^{\lambda} s_{k}(\alpha \sigma)^{-1}=x_{\alpha(i) \alpha(j)}^{\lambda}
$$

par définition des $x_{i j}^{\lambda}$, car $(\alpha \sigma)(k)=\alpha(i)$ et $(\alpha \sigma)(k+1)=\alpha(j)$. 
Vérifions enfin les relations de Steinberg.

(S.1) Pour la première, en utilisant la première des relations (1), l'on a

$$
x_{i j}^{\lambda} x_{i j}^{\mu}=\sigma y_{k}^{\lambda} s_{k} \sigma^{-1} \sigma y_{k}^{\mu} s_{k} \sigma^{-1}=\sigma y_{k}^{\lambda} y_{k}^{0} y_{k}^{\mu} s_{k} \sigma^{-1}=\sigma y_{k}^{\lambda+\mu} s_{k} \sigma^{-1}=x_{i j}^{\lambda+\mu} .
$$

(S.2) Pour la seconde relation de Steinberg, il suffit de vérifier que $x_{12}^{\lambda} x_{34}^{\mu}=x_{34}^{\mu} x_{12}^{\lambda}$ et que $x_{12}^{\lambda} x_{13}^{\mu}=x_{13}^{\mu} x_{12}^{\lambda}$. La première égalité s'obtient clairement de la deuxième relation (1) puisque $x_{12}^{\lambda}=y_{1}^{\lambda} y_{1}^{0}$ et $x_{34}^{\mu}=y_{3}^{\mu} y_{3}^{0}$. Pour la seconde, on a, en utilisant plusieurs fois la relation de tresse dans (1),

$$
\begin{aligned}
x_{12}^{\lambda} x_{13}^{\mu} & =y_{1}^{\lambda} s_{1} s_{2} y_{1}^{\mu} s_{1} s_{2} \\
& =y_{1}^{\lambda} \underbrace{y_{1}^{0} y_{2}^{0} y_{1}^{\mu}} y_{1}^{0} y_{2}^{0} \\
& =\underbrace{y_{1}^{\lambda} y_{2}^{\mu} y_{1}^{0}} \underbrace{y_{2}^{0} y_{1}^{0} y_{2}^{0}} \\
& =y_{2}^{0} y_{1}^{\mu} \underbrace{y_{2}^{\lambda} y_{1}^{0} y_{2}^{0}} y_{1}^{0} \\
& =y_{2}^{0} y_{1}^{\mu} y_{1}^{0} y_{2}^{0} y_{1}^{\lambda} y_{1}^{0} \\
& =\underbrace{s_{2} y_{1}^{\mu} s_{1} s_{2}} \underbrace{y_{1}^{\lambda} s_{1}} \\
& =x_{13}^{\mu} x_{12}^{\lambda} .
\end{aligned}
$$

(S.3) Enfin, la dernière relation de Steinberg se vérifie comme suit en utilisant (S) et plusieurs fois la relation $y_{i}^{0} y_{i}^{0}=1$ :

$$
\begin{aligned}
x_{12}^{\lambda} x_{23}^{\mu} & =y_{1}^{\lambda} y_{1}^{0} y_{2}^{\mu} y_{2}^{0} \\
& =y_{1}^{\lambda} \underbrace{y_{1}^{0} y_{2}^{\mu} y_{1}^{0}} y_{1}^{0} y_{2}^{0} \\
& =\underbrace{y_{1}^{\lambda} y_{2}^{0} y_{1}^{\mu}} y_{2}^{0} y_{1}^{0} y_{2}^{0} \\
& =y_{2}^{\mu} y_{1}^{\lambda \mu} y_{2}^{\lambda} y_{2}^{0} y_{1}^{0} y_{2}^{0} \\
& =\underbrace{y_{2}^{\mu} y_{2}^{0}} \underbrace{y_{2}^{0} y_{1}^{\lambda \mu} y_{2}^{\lambda}} y_{2}^{0} y_{1}^{0} y_{2}^{0} \\
& =x_{23}^{\mu} y_{1}^{\lambda} y_{2}^{\lambda \mu} y_{1}^{0} y_{2}^{0} y_{1}^{0} y_{2}^{0} \\
& =x_{23}^{\mu} \underbrace{y_{1}^{\lambda} y_{1}^{0}} y_{1}^{0} y_{2}^{\lambda \mu} \underbrace{y_{1}^{0} y_{2}^{0} y_{1}^{0}} y_{2}^{0} \\
& =x_{23}^{\mu} x_{12}^{\lambda} y_{1}^{0} y_{2}^{\lambda \mu} y_{2}^{0} y_{1}^{0} \underbrace{y_{2}^{0} y_{2}^{0}} \\
& =x_{23}^{\mu} x_{12}^{\lambda} \underbrace{y_{1}^{0} y_{2}^{\lambda \mu} y_{2}^{0} y_{1}^{0}} \\
& =x_{23}^{\mu} x_{12}^{\lambda} x_{13}^{\lambda \mu} .
\end{aligned}
$$

Les calculs précédents montrent que $\chi\left(x_{i, i+1}^{\lambda}\right)=y_{i}^{\lambda} y_{i}^{0}$ et $\chi\left(s_{i}\right)=y_{i}^{0} \in G_{n}(\Lambda)$ définissent un homomorphisme de groupes $\chi: G_{n}(\Lambda) \rightarrow H$ qui est clairement inverse de $\psi$. 


\section{Stabilisation}

Soit $G L(\Lambda)$ (resp. $\mathfrak{S}_{\infty}$ ) la limite inductive des groupes $G L_{n}(\Lambda)$ (resp. des groupes symétriques $\mathfrak{S}_{n}$ ) suivant l'injection standard $G L_{n}(\Lambda) \rightarrow G L_{n+1}(\Lambda)$ (resp. $\mathfrak{S}_{n} \rightarrow \mathfrak{S}_{n+1}$ ). On considère aussi le groupe de Steinberg stabilisé $S t(\Lambda)$ qui est la limite inductive des groupes $S t_{n}(\Lambda)$. L'homomorphisme $\varphi$ du paragraphe 1 s'étend en un homomorphisme $\varphi: S t(\Lambda) \rightarrow G L(\Lambda)$ dont l'image est le sous-groupe $E(\Lambda)$ engendré par les matrices élémentaires. Le noyau de $\varphi$ est le groupe de K-théorie algébrique $K_{2}(\Lambda)$ introduit par Milnor (voir [3], Chap. 5).

Le groupe $\mathfrak{S}_{\infty}$ opère sur $S t(\Lambda)$, ce qui permet de définir le produit semi-direct $G(\Lambda)$ de $\mathfrak{S}_{\infty}$ par $S t(\Lambda)$. Il est clair que $G(\Lambda)$ est la limite inductive des groupes $G_{n}(\Lambda)$. De même que $S t(\Lambda)$ est engendré par les symboles $x_{i j}^{\lambda}$ où $i$ et $j$ parcourent tous les entiers distincts $\geq 1$, le groupe stabilisé $G(\Lambda)$ admet la présentation donnée dans le théorème 1 avec pour générateurs les symboles $y_{i}^{\lambda}$ où $i$ parcourt maintenant tous les entiers $\geq 1$.

L'homomorphisme $\Phi$ du paragraphe 1 s'étend en un homomorphisme de groupes $\Phi$ : $G(\Lambda) \rightarrow G L(\Lambda)$. Son image $E S(\Lambda)$ est le sous-groupe de $G L(\Lambda)$ engendré par $E(\Lambda)$ et les matrices de permutation.

Lemme 2.- Soit $S$ le sous-groupe de $\mathfrak{S}_{\infty}$ formé des matrices de permutation appartenant $\grave{a} E(\Lambda)$. S'il existe une matrice de permutation impaire dans $E(\Lambda)$, alors toutes les matrices de permutation sont dans $E(\Lambda)$ et l'on a $E S(\Lambda)=E(\Lambda)$ et $S=\mathfrak{S}_{\infty}$. Sinon, $E(\Lambda)$ est un sous-groupe d'indice deux dans $E S(\Lambda)$ et $S$ s'identifie au sous-groupe $\mathfrak{A}_{\infty}$ des permutations paires de $\mathfrak{S}_{\infty}$.

DÉMOnStration.- On sait que toute matrice de permutation paire appartient à $E(\mathbf{Z})$, donc à $E(\Lambda)\left(c f\right.$. [3], Chap. 9). Par conséquent, $\mathfrak{A}_{\infty} \subset S \subset \mathfrak{S}_{\infty}$. Comme $\mathfrak{A}_{\infty}$ est d'indice deux dans $\mathfrak{S}_{\infty}$, on a soit $S=\mathfrak{S}_{\infty}$, soit $S=\mathfrak{A}_{\infty}$.

Nous dirons que l'anneau $\Lambda$ est normal si $E S(\Lambda)$ contient $E(\Lambda)$ comme sous-groupe d'indice deux. Dans le cas où $E S(\Lambda)=E(\Lambda)$, nous dirons qu'il est pathologique. Parmi les anneaux normaux l'on trouve les anneaux $\Lambda$ munis d'un morphisme $\varphi: \Lambda \rightarrow \Lambda^{\prime}$ vers un anneau commutatif $\Lambda^{\prime}$ dans lequel $-1 \neq 1$. En effet, si $\sigma$ est la matrice d'une transposition dans $G L(\Lambda)$, on a $\operatorname{det}(\varphi(\sigma))=-1$. Il en résulte qu'une algèbre de groupe ou une algèbre de polynômes à coefficients dans $\mathbf{Z}$ ou $\mathbf{Z} / n$ avec $n>2$ est normale. Par contre, une $\mathbf{Z} / 2$-algèbre est pathologique. L'anneau $\mathbf{Z} / 4<a, b\rangle /(a b-2, b a)$ est un exemple d'anneau pathologique qui n'est pas une $\mathbf{Z} / 2$-algèbre.

Soit $N^{\prime}(\Lambda)$ le noyau de l'homomorphisme $\Phi: G(\Lambda) \rightarrow G L(\Lambda)$.

Proposition 1.- Le groupe $N^{\prime}(\Lambda)$ est le centralisateur de $\operatorname{St}(\Lambda)$ dans $G(\Lambda)$.

Démonstration.- Elle est inspirée de celle du théorème 5.1 de [3]. Si $x$ est un élément du centralisateur, alors la matrice $\Phi(x)$ commute à toutes les matrices élémentaires $e_{i j}^{\lambda}$. Donc $\Phi(x)$ est une matrice scalaire. On conclut en remarquant qu'une matrice scalaire de $G L(\Lambda)$ est nécessairement la matrice identité. Ainsi $x \in N^{\prime}(\Lambda)$.

Réciproquement, soit $y \in G(\Lambda)$ tel que $\Phi(y)=1$. Il suffit de montrer que $y$ commute à tous les générateurs $x_{i j}^{\lambda}$ du groupe de Steinberg. Soit $n$ assez grand pour que $y$ soit le produit d'éléments $x_{i j}^{\lambda}$ avec $i, j<n$ et d'une permutation $\sigma \in \mathfrak{S}_{n-1}$. Soit $P_{n}$ le sousgroupe de $S t(\Lambda)$ engendré par $x_{1 n}^{\mu_{1}}, x_{2 n}^{\mu_{2}}, \ldots, x_{n-1, n}^{\mu_{n-1}}$ où $\mu_{1}, \mu_{2}, \ldots, \mu_{n-1} \in \Lambda$. D'après le 
lemme 5.2 de [3], le sous-groupe $P_{n}$ est commutatif et la restriction de $\Phi$ à $P_{n}$ est injective ; de plus, $x_{i j}^{\lambda} P_{n} x_{i j}^{-\lambda} \subset P_{n}$ si $i<n$ et $j<n$. De même si $\sigma \in \mathfrak{S}_{n-1}$, on a $\sigma P_{n} \sigma^{-1} \subset P_{n}$ car $\sigma$ permute $\{1,2, \ldots, n-1\}$ et fixe $n$. Par conséquent, $y P_{n} y^{-1} \subset P_{n}$.

Pour tout $x \in P_{n}$, on a $y x y^{-1} \in P_{n}$ d'après ce qui précède. Les égalités

$$
\Phi\left(y x y^{-1}\right)=\Phi(y) \Phi(x) \Phi(y)^{-1}=\Phi(x)
$$

montrent que $y x y^{-1}=x$ puisque la restriction de $\Phi$ à $P_{n}$ est injective. Il en résulte que $y$ commute aux éléments $x_{k n}^{\mu}$ avec $k<n$. On montrerait de même que $y$ commute aux éléments $x_{n \ell}^{\mu}$ avec $\ell<n$. Par conséquent, $y$ commute à $x_{k n}^{\mu} x_{n \ell}^{1} x_{k n}^{-\mu} x_{n \ell}^{-1}=x_{k \ell}^{\mu}$. On conclut en prenant $n$ arbitrairement grand.

Corollaire 1.- On a une extension centrale de groupes

$$
0 \rightarrow K_{2}(\Lambda) \rightarrow N^{\prime}(\Lambda) \stackrel{\pi}{\longrightarrow} S \rightarrow 1
$$

où $\pi$ est la composée de l'inclusion de $N^{\prime}(\Lambda)$ dans $G(\Lambda)$ et de la surjection canonique de $G(\Lambda)$ sur $\mathfrak{S}_{\infty}=G(\Lambda) / S t(\Lambda)$.

DÉmonstration. - Montrons d'abord que la suite précédente est exacte. L'image de $\pi$ est $S$. En effet, soit $x=w \sigma$ un élément de $N^{\prime}(\Lambda)$ avec $w \in S t(\Lambda)$ et $\sigma=\pi(x) \in \mathfrak{S}_{\infty}$. Par définition de $N^{\prime}(\Lambda)$ on a $1=\Phi(x)=\varphi(w) \sigma$ où, comme précédemment, l'on identifie $\sigma$ avec la matrice de permutation correspondante. On voit ainsi que $\pi(x)=\sigma=\varphi(w)^{-1}$ appartient à la fois à $\mathfrak{S}_{\infty}$ et à l'image de $\varphi$, donc à $S$. Réciproquement, soit $\sigma \in S=$ $E(\Lambda) \bigcap \mathfrak{S}_{\infty}$. Comme l'image de $\varphi$ est $E(\Lambda)$, il existe un élément $w$ de $S t(\Lambda)$ tel que $\varphi(w)=\sigma^{-1}$. Par suite, $w \sigma$ appartient à $N^{\prime}(\Lambda)$ et $\pi(w \sigma)=\sigma$.

Déterminons le noyau de $\pi: N^{\prime}(\Lambda) \rightarrow S$. Soit $x=w \sigma$ un élément du noyau avec $w \in S t(\Lambda)$ et $\sigma=\pi(x) \in \mathfrak{S}_{\infty}$. On a $\pi(x)=1$ et

$$
1=\Phi(x)=\varphi(w) \sigma=\varphi(w) \pi(x)=\varphi(w)
$$

Donc $x=w$ avec $\varphi(w)=1$. Il appartient donc au noyau de $\varphi$, c'est-à-dire à $K_{2}(\Lambda)$. Réciproquement, si $x=w$ avec $w \in K_{2}(\Lambda)$, alors $\pi(x)=1$ et $\Phi(x)=\varphi(w) \pi(x)=1$.

D'après la proposition 1 , tout élément de $N^{\prime}(\Lambda)$ commute à tout élément du groupe de Steinberg et donc à tout élément du sous-groupe $K_{2}(\Lambda)$. Il en résulte que l'extension est centrale.

Nous considérons maintenant le sous-groupe $N(\Lambda)$ constitué des éléments $x$ de $N^{\prime}(\Lambda)$ tels que $\pi(x)$ appartient à $\mathfrak{A}_{\infty}$. Il est clair que $N(\Lambda)=N^{\prime}(\Lambda)$ si $\Lambda$ est un anneau normal. Si $\Lambda$ est pathologique, alors $N(\Lambda)$ est un sous-groupe d'indice deux de $N^{\prime}(\Lambda)$.

Le reste de cet article est consacré à la détermination de la structure du groupe $N(\Lambda)$ (c'est-à-dire du noyau de $\Phi: G(\Lambda) \rightarrow G L(\Lambda)$ si $\Lambda$ est normal).

Si l'on restreint l'extension du corollaire 1 à $\mathfrak{A}_{\infty}$, on a le résultat suivant.

Corollaire 2.-L'extension $0 \rightarrow K_{2}(\Lambda) \rightarrow N(\Lambda) \stackrel{\pi}{\longrightarrow} \mathfrak{A}_{\infty} \rightarrow 1$ est centrale. 
On sait depuis Schur [5] (voir aussi [2], Théorème 2.12.5) que le groupe alterné $\mathfrak{A}_{n}$ d'ordre $n$ a une extension centrale universelle de noyau $C \cong \mathbf{Z} / 2$ dès que $n>7$. Il en est de même de $\mathfrak{A}_{\infty}$ dont l'extension centrale universelle sera notée $\widetilde{\mathfrak{A}}_{\infty}$. L'universalité de $\widetilde{\mathfrak{A}}_{\infty}$ et le corollaire 2 appliqué à l'anneau $\mathbf{Z}$ des entiers relatifs impliquent que l'on a un unique morphisme d'extension centrales

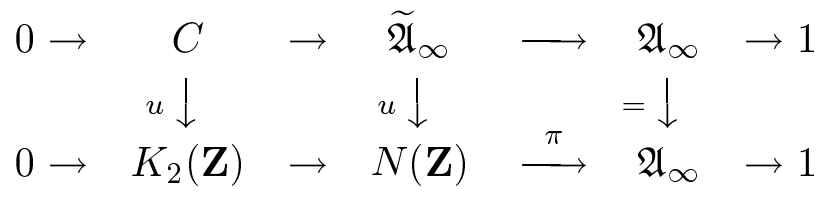

Comme $K_{2}(\mathbf{Z}) \cong \mathbf{Z} / 2$ (cf. [3], Corollaire 10.2), la question se pose de savoir si l'application $u: C \rightarrow K_{2}(\mathbf{Z})$ ainsi définie est nulle ou non. La réponse est donnée au théorème suivant.

ThÉORÈme 2.- Les morphismes $u: C \rightarrow K_{2}(\mathbf{Z})$ et $u: \widetilde{\mathfrak{A}}_{\infty} \rightarrow N(\mathbf{Z})$ du diagramme (4) sont des isomorphismes.

Ce théorème sera démontré au paragraphe 4. Nous l'utilisons pour déterminer la structure du groupe $N(\Lambda)$ pour un anneau $\Lambda$ général.

ThÉORÈme 3.- Soit $i: K_{2}(\mathbf{Z}) \rightarrow K_{2}(\Lambda)$ l'application induite par le morphisme canonique $\mathbf{Z} \rightarrow \Lambda$. On a l'isomorphisme de groupes

$$
N(\Lambda) \cong\left(\widetilde{\mathfrak{A}}_{\infty} \times K_{2}(\Lambda)\right) /<\left(u^{-1}(\gamma), i(\gamma)\right)>
$$

où $\gamma$ est le générateur de $K_{2}(\mathbf{Z})$ et $\left\langle\left(u^{-1}(\gamma), i(\gamma)\right)>\right.$ est le sous-groupe d'ordre deux engendré par l'élément central $\left(u^{-1}(\gamma), i(\gamma)\right)$ de $\widetilde{\mathfrak{A}}_{\infty} \times K_{2}(\Lambda)$. En particulier, si l'application $i: K_{2}(\mathbf{Z}) \rightarrow K_{2}(\Lambda)$ est nulle, alors

$$
N(\Lambda) \cong \mathfrak{A}_{\infty} \times K_{2}(\Lambda)
$$

Si $\Lambda$ est un anneau augmenté (autrement dit, s'il est muni d'un morphisme d'anneaux $\varepsilon: \Lambda \rightarrow \mathbf{Z})$, on $a$

$$
N(\Lambda) \cong \widetilde{\mathfrak{A}}_{\infty} \times K_{2}(\Lambda) / i\left(K_{2}(\mathbf{Z})\right)
$$

DÉmonstration. - Le théorème 2 implique que l'extension centrale $N(\mathbf{Z}) \stackrel{\pi}{\longrightarrow} \mathfrak{A}_{\infty}$ est universelle. D'après le corollaire 2 il existe donc un unique morphisme d'extensions centrales

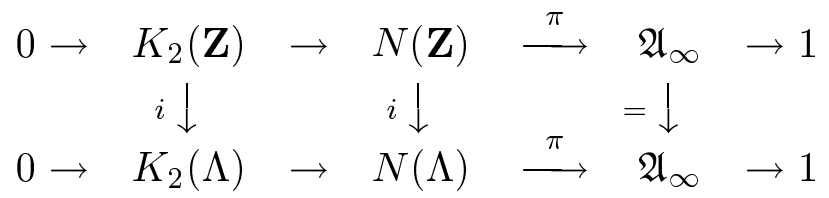

Puisqu'il est unique, $i$ est nécessairement induit par le morphisme canonique $\mathbf{Z} \rightarrow \Lambda$.

a) Soit $M=N(\mathbf{Z}) \times K_{2}(\Lambda)$. Comme $K_{2}(\mathbf{Z})$ est abélien, $x \mapsto\left(x, i(x)^{-1}\right)$ définit un homomorphisme de groupes $\delta: K_{2}(\mathbf{Z}) \rightarrow M$. On voit immédiatement que $\delta$ est injectif et que son image est un sous-groupe distingué de $M$. 
Pour tout $(x, y) \in M$, posons $f(x, y)=i(x) y \in N(\Lambda)$. Comme $K_{2}(\Lambda)$ est central dans $N(\Lambda)$, l'application $f$ est un homomorphisme de groupes. Si $x \in K_{2}(\mathbf{Z})$, on a

$$
(f \circ \delta)(x)=f\left(x, i(x)^{-1}\right)=i(x) i(x)^{-1}=1,
$$

ce qui permet de définir un homomorphisme de groupes $f^{\prime}: M / \delta\left(K_{2}(\mathbf{Z})\right) \rightarrow N(\Lambda)$ par

$$
f^{\prime}\left((x, y) \bmod \delta\left(K_{2}(\mathbf{Z})\right)\right)=i(x) y \text {. }
$$

Considérons l'homomorphisme composé $\pi \circ f^{\prime}: M \rightarrow \mathfrak{A}_{\infty}$. Montrons d'abord qu'il est surjectif. En effet, si $\sigma \in \mathfrak{A}_{\infty}$, il existe $x \in N(\mathbf{Z})$ tel que $\pi(x)=\sigma$. Par conséquent,

$$
\left(\pi \circ f^{\prime}\right)\left((x, 1) \bmod \delta\left(K_{2}(\mathbf{Z})\right)\right)=\pi(i(x))=\pi(x)=\sigma .
$$

Etablissons maintenant que le noyau de $\pi \circ f^{\prime}$ est isomorphe à $K_{2}(\Lambda)$. Soit $(x, y) \in M$ tel que $\pi(i(x) y)=1$. Alors il existe $z \in K_{2}(\Lambda)$ tel que $i(x) y=z$. Il en résulte que dans $M$ on a

$$
(x, y) \equiv(x, y)\left(x^{-1}, i(x)\right)=(x, y)\left(x^{-1}, z y^{-1}\right)=\left(1, y z y^{-1}\right)
$$

modulo $\delta\left(K_{2}(\mathbf{Z})\right)$, ce qui prouve que tout élément du noyau de $\pi \circ f^{\prime}$ provient de $K_{2}(\Lambda)$. Il reste à voir que l'homomorphisme $z \mapsto(1, z) \bmod \delta\left(K_{2}(\mathbf{Z})\right)$ de $K_{2}(\Lambda)$ dans $M / \delta\left(K_{2}(\mathbf{Z})\right)$ est injectif. Mais si $(1, z)=\left(x, i(x)^{-1}\right)$, alors $x=1$ et donc $z=i(x)^{-1}=1$.

Nous avons ainsi construit un diagramme commutatif de suites exactes

$$
\begin{aligned}
& 0 \rightarrow K_{2}(\Lambda) \rightarrow M / \delta\left(K_{2}(\mathbf{Z})\right) \stackrel{\pi \circ f^{\prime}}{\longrightarrow} \mathfrak{A}_{\infty} \rightarrow 1 \\
& f^{\prime} \downarrow \quad=\downarrow \\
& 0 \rightarrow K_{2}(\Lambda) \rightarrow N(\Lambda) \quad \stackrel{\pi}{\longrightarrow} \mathfrak{A}_{\infty} \rightarrow 1
\end{aligned}
$$

On vérifie sans peine que $f^{\prime}$ induit l'identité sur les noyaux. Il en résulte que $f^{\prime}$ est un isomorphisme. On conclut à l'aide du théorème 2.

b) Si $i: K_{2}(\mathbf{Z}) \rightarrow K_{2}(\Lambda)$ est nulle, alors $\delta(x)=(x, 1)$, et donc d'après (a)

$$
N(\Lambda) \cong M / \delta\left(K_{2}(\mathbf{Z})\right) \cong\left(N(\mathbf{Z}) / K_{2}(\mathbf{Z})\right) \times K_{2}(\Lambda) \cong \mathfrak{A}_{\infty} \times K_{2}(\Lambda) .
$$

c) Si $\Lambda$ est un anneau augmenté, l'on a par fonctorialité le diagramme commutatif

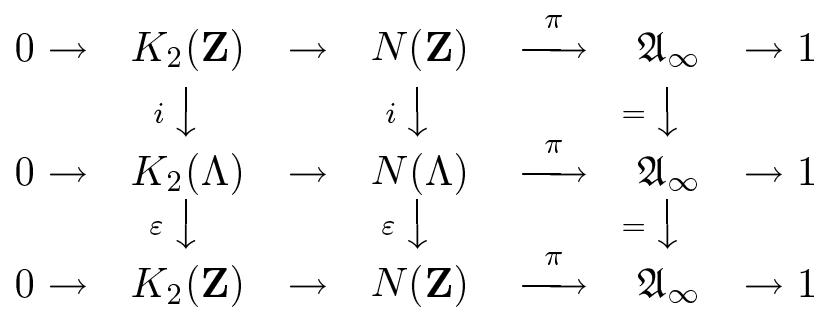

Ceci montre que le groupe abélien $K_{2}(\Lambda)$ se met sous la forme $K_{2}(\Lambda)=K_{2}(\mathbf{Z}) \oplus \widetilde{K}_{2}(\Lambda)$ et que le noyau de $\varepsilon: N(\Lambda) \rightarrow N(\mathbf{Z})$ est $\widetilde{K}_{2}(\Lambda)$. Comme le morphisme $\varepsilon$ est scindé par $i$ et que $\widetilde{K}_{2}(\Lambda) \subset K_{2}(\Lambda)$ est central dans $N(\Lambda)$, l'extension centrale

$$
0 \rightarrow \widetilde{K}_{2}(\Lambda) \rightarrow N(\Lambda) \stackrel{\varepsilon}{\longrightarrow} N(\mathbf{Z}) \rightarrow 1
$$

est scindée. Par conséquent, $N(\Lambda) \cong N(\mathbf{Z}) \times \widetilde{K}_{2}(\Lambda)$. On conclut à nouveau à l'aide $d u$ théorème 2 . 


\section{Quelques calculs dans des extensions centrales de groupes}

Soit $0 \rightarrow C \rightarrow S \rightarrow E \rightarrow 1$ une extension centrale de groupes. Si deux éléments $\alpha$ et $\beta$ du groupe $E$ commutent, c'est-à-dire si leur commutateur $[\alpha, \beta]=\alpha \beta \alpha^{-1} \beta^{-1}$ vaut 1 , ils définissent un élément $\alpha * \beta$ du noyau $C$ par la formule

$$
\alpha * \beta=[x, y]
$$

où $x$ et $y$ sont des relèvements quelconques dans $S$ de $\alpha$ et de $\beta$ respectivement (voir $[3$, Chap. 8).

Appliquons cette construction aux permutations paires $\alpha=(12)(34)$ et $\beta=(13)(24)$. Comme elles commutent, elles définissent un élément $\alpha *_{A} \beta$ du noyau $C \cong \mathbf{Z} / 2$ de l'extension centrale $\widetilde{\mathfrak{A}}_{\infty} \rightarrow \mathfrak{A}_{\infty}$.

Considérons maintenant l'extension centrale $0 \rightarrow K_{2}(\mathbf{Z}) \rightarrow N(\mathbf{Z}) \stackrel{\pi}{\longrightarrow} \mathfrak{A}_{\infty} \rightarrow 1 \mathrm{du}$ corollaire 2. La construction (5) fournit un élément $\alpha *_{N} \beta$ du noyau $K_{2}(\mathbf{Z})$. Il est clair que $\alpha *_{N} \beta$ est l'image de $\alpha *_{A} \beta$ par l'homomorphisme $u$ du diagramme (4).

DÉmonstration Du thÉorème 2.- Au vu de ce qui précède, il suffit de vérifier que l'élément central $\alpha *_{N} \beta$ de $N(\mathbf{Z})$ n'est pas trivial. Pour le calculer, il faut tout d'abord relever $\alpha$ et $\beta$ dans $N(\mathbf{Z})$. Pour tout $i \neq j$ posons $w_{i j}=x_{i j}^{1} x_{j i}^{-1} x_{i j}^{1}$ et $H_{i j}=w_{i j}^{-2}$ (l'élément $H_{i j}$ est noté $h_{i j}(-1)$ dans [3], Chap. 9). Observons qu'une permutation $\sigma$ opère sur $w_{i j}$ et sur $H_{i j}$ par ${ }^{\sigma} w_{i j}=w_{\sigma(i) \sigma(j)}$ et ${ }^{\sigma} H_{i j}=H_{\sigma(i) \sigma(j)}$. Définissons

$$
A=w_{12} w_{34} H_{13}^{-1} \quad \text { et } \quad B=w_{13} w_{24} H_{12}^{-1}
$$

dans le groupe de Steinberg $S t(\mathbf{Z})$. On a

$$
\varphi(A)=\widehat{\alpha}=\left(\begin{array}{llll}
0 & 1 & 0 & 0 \\
1 & 0 & 0 & 0 \\
0 & 0 & 0 & 1 \\
0 & 0 & 1 & 0
\end{array}\right) \quad \text { et } \quad \varphi(B)=\widehat{\beta}=\left(\begin{array}{llll}
0 & 0 & 1 & 0 \\
0 & 0 & 0 & 1 \\
1 & 0 & 0 & 0 \\
0 & 1 & 0 & 0
\end{array}\right) .
$$

Les matrices $\widehat{\alpha}$ et $\widehat{\beta}$ sont les matrices des permutations $\alpha$ et $\beta$ respectivement. Nous affirmons que l'élément $A^{-1} \alpha$ (resp. l'élément $B^{-1} \beta$ ) de $G(\mathbf{Z})$ est un relèvement de $\alpha$ (resp. de $\beta$ ) dans $N(\mathbf{Z})$. En effet,

$$
\Phi\left(A^{-1} \alpha\right)=\varphi(A)^{-1} \widehat{\alpha}=\widehat{\alpha}^{-1} \widehat{\alpha}=1 \quad \text { et } \quad \pi\left(A^{-1} \alpha\right)=\alpha .
$$

De même pour $B^{-1} \beta$.

L'élément $\alpha *_{N} \beta$ du noyau $K_{2}(\mathbf{Z})$ de l'extension centrale $N(\mathbf{Z}) \stackrel{\pi}{\longrightarrow} \mathfrak{A}_{\infty}$ se calcule donc comme suit :

$$
\begin{aligned}
\alpha *_{N} \beta & =\left[A^{-1} \alpha, B^{-1} \beta\right] \\
& =A^{-1} \alpha B^{-1} \beta \alpha^{-1} A \beta^{-1} B \\
& =A^{-1} \alpha B^{-1} \alpha^{-1} \beta A \beta^{-1} B \\
& =A^{-1} \alpha\left(B^{-1}\right){ }^{\beta} A B
\end{aligned}
$$


car $\alpha$ et $\beta$ commutent. En remplaçant $A$ et $B$ par leur valeur, on a

$$
\alpha *_{N} \beta=H_{13} w_{34}^{-1} w_{12}^{-1} H_{21} w_{13}^{-1} w_{24}^{-1} w_{34} w_{12} H_{31}^{-1} w_{13} w_{24} H_{12}^{-1}
$$

Le corollaire 9.4 de [3] implique que $w_{13}$ et $w_{24}$ commutent. Par ailleurs,

$$
\varphi\left(w_{13}^{-1} w_{24}^{-1}\right)=\left(\begin{array}{cccc}
0 & 0 & 1 & 0 \\
0 & 0 & 0 & 1 \\
1 & 0 & 0 & 0 \\
0 & 1 & 0 & 0
\end{array}\right)\left(\begin{array}{cccc}
1 & 0 & 0 & 0 \\
0 & 1 & 0 & 0 \\
0 & 0 & -1 & 0 \\
0 & 0 & 0 & -1
\end{array}\right) .
$$

Une autre application de loc. cit. montre alors que

$$
w_{13}^{-1} w_{24}^{-1} w_{34} w_{12} H_{31}^{-1} w_{13} w_{24}=w_{12} w_{34} H_{13}^{-1}
$$

ce qui nous permet d'écrire

$$
\begin{aligned}
\alpha *_{N} \beta & =H_{13} w_{34}^{-1} w_{12}^{-1} H_{21} \underbrace{w_{13}^{-1} w_{24}^{-1} w_{34} w_{12} H_{31}^{-1} w_{13} w_{24}} H_{12}^{-1} \\
& =H_{13} w_{34}^{-1} w_{12}^{-1} H_{21} w_{12} w_{34} H_{13}^{-1} H_{12}^{-1} .
\end{aligned}
$$

Toujours d'après le corollaire 9.4 de [3], les éléments $w_{12}$ et $w_{34}$ commutent également et

$$
\varphi\left(w_{12}^{-1} w_{34}^{-1}\right)=\left(\begin{array}{cccc}
0 & 1 & 0 & 0 \\
1 & 0 & 0 & 0 \\
0 & 0 & 0 & 1 \\
0 & 0 & 1 & 0
\end{array}\right)\left(\begin{array}{cccc}
1 & 0 & 0 & 0 \\
0 & -1 & 0 & 0 \\
0 & 0 & 1 & 0 \\
0 & 0 & 0 & -1
\end{array}\right)
$$

Par conséquent, $w_{34}^{-1} w_{12}^{-1} H_{21} w_{12} w_{34}=H_{12}$, d'où nous tirons

$$
\begin{aligned}
\alpha *_{N} \beta & =H_{13} \underbrace{w_{34}^{-1} w_{12}^{-1} H_{21} w_{12} w_{34}} H_{13}^{-1} H_{12}^{-1} \\
& =H_{13} H_{12} H_{13}^{-1} H_{12}^{-1} \\
& =\left[H_{12}, H_{13}\right]^{-1}
\end{aligned}
$$

qui n'est autre, d'après Milnor (voir [3], Chap. 8-10), que le générateur de $K_{2}(\mathbf{Z})$.

Corollaire 3.- L'élément $\alpha *_{A} \beta$ est le générateur du noyau $C \cong \mathbf{Z} / 2$ de l'extension centrale universelle $\widetilde{\mathfrak{A}}_{\infty} \rightarrow \mathfrak{A}_{\infty}$.

Nous terminons en donnant une expression du générateur du groupe $K_{2}(\mathbf{Z})$ à l'aide des matrices de permutation $\widehat{\alpha}$ et $\widehat{\beta}$ de $(7)$. Ces matrices appartiennent à $E(\mathbf{Z})$. Par la construction (5) elles définissent donc un élément $\widehat{\alpha} *_{S t} \widehat{\beta}$ du noyau $K_{2}(\mathbf{Z})$ de l'extension centrale $S t(\mathbf{Z}) \rightarrow E(\mathbf{Z})$. 
Proposition 2.-L'élément $\widehat{\alpha} *_{S t} \widehat{\beta}$ engendre le groupe $K_{2}(\mathbf{Z})$.

Démonstration.- Soit $A$ et $B$ les éléments de $S t(\mathbf{Z})$ définis par (6). Comme ils relèvent $\widehat{\alpha}$ et $\widehat{\beta}$, on a $\widehat{\alpha} *_{S t} \widehat{\beta}=[A, B]$. Un calcul similaire à celui de la démonstration du théorème 2 permettrait de montrer que le commutateur $[A, B]$ est le générateur de $K_{2}(\mathbf{Z})$.

Nous allons procéder différemment. Rappelons (voir [3], Chap. Ê7-10) qu'il existe un isomorphisme naturel de $K_{2}(\mathbf{Z})$ vers le groupe fondamental $\pi_{1} S L(\mathbf{R}) \cong \mathbf{Z} / 2$ où $\mathbf{R}$ est le corps des nombres réels et où $S L(\mathbf{R})$ est le sous-groupe des éléments de $G L(\mathbf{R})$ de déterminant 1. Il suffit donc de montrer que l'image de $\widehat{\alpha} *_{S t} \widehat{\beta}$ dans le groupe $\pi_{1} S L(\mathbf{R}) \cong$ $\pi_{1} S L_{4}(\mathbf{R})$ n'est pas triviale. Pour établir ce dernier point, nous allons montrer que $\widehat{\alpha} *_{S t} \widehat{\beta}$ s'envoie sur le générateur du centre du groupe de revêtement universel du groupe $S O(4)$ des matrices spéciales orthogonales, ce dernier étant un rétract par déformation de $S L_{4}(\mathbf{R})$.

Soit $\mathbf{H}$ le corps des quaternions muni de la structure euclidienne induite par la norme $\|x\|=\left(x x^{*}\right)^{1 / 2}$ où $x^{*}$ désigne le conjugué de $x$. Notons $S^{3}$ le groupe multiplicatif des quaternions de norme 1 . Si $u$ et $v$ sont des éléments de $S^{3}$, l'application linéaire $x \mapsto u x v^{*}$ est une isométrie positive $\rho(u, v)$ de $\mathbf{H}$. Dans la base orthonormée $(1, i, j, k)$ formée par les générateurs standard de $\mathbf{H}$, on peut identifier $\rho(u, v)$ à un élément de $S O(4)$. Ceci définit un homomorphisme surjectif de groupes $\rho: S^{3} \times S^{3} \rightarrow S O(4)$ dont le noyau, d'ordre deux, est engendré par $(-1,-1)$. Il est bien connu que cette extension centrale est le groupe de revêtement universel de $S O(4)$ (voir par exemple [4], Chap. 10).

Revenons aux matrices $\widehat{\alpha}$ et $\widehat{\beta}$. Le calcul montre que $\alpha=\rho(k, j)$ et $\beta=\rho(i, k)$. Par conséquent, $\widehat{\alpha} *_{S t} \widehat{\beta}$ s'envoie sur le commutateur

$$
\kappa=(k, j)(i, k)(k, j)^{-1}(i, k)^{-1} \in S^{3} \times S^{3} .
$$

On conclut en observant que dans le corps de quaternions l'on a

$$
\kappa=\left(k i k^{*} i^{*}, j k j^{*} k^{*}\right)=(-1,-1) .
$$

Corollaire 4.- L'extension centrale universelle $\widetilde{\mathfrak{A}}_{\infty}$ de $\mathfrak{A}_{\infty}$ est isomorphe au sousgroupe de $\operatorname{St}(\mathbf{Z})$ formé des éléments dont l'image par $\varphi$ est une matrice de permutation paire :

$$
\widetilde{\mathfrak{A}}_{\infty} \cong\left\{w \in S t(\mathbf{Z}) \mid \varphi(w) \in \mathfrak{A}_{\infty}\right\} .
$$

Démonstration.- Par universalité de $\widetilde{\mathfrak{A}}_{\infty}$, l'inclusion des matrices de permutation paires dans $E(\mathbf{Z})$ induit un unique morphisme d'extensions de groupes

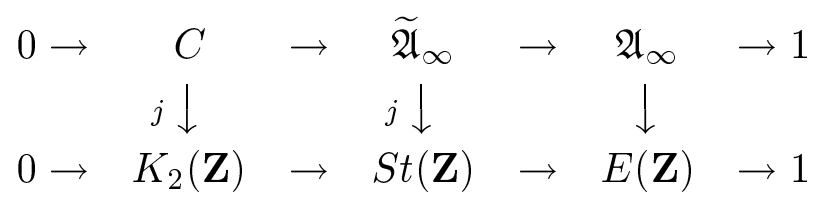

Par construction, $j$ envoie le générateur $\alpha *_{A} \beta$ de $C$ sur l'élément $\widehat{\alpha} *_{S t} \widehat{\beta}$ dont nous venons de montrer que c'est le générateur de $K_{2}(\mathbf{Z})$. Il en résulte que $j: C \rightarrow K_{2}(\mathbf{Z})$ est un isomorphisme. Ceci, joint à la commutativité du diagramme (8), implique que l'homomorphisme $j: \widetilde{\mathfrak{A}}_{\infty} \rightarrow S t(\mathbf{Z})$ induit un isomorphisme de $\widetilde{\mathfrak{A}}_{\infty}$ sur le sous-groupe des éléments dont l'image par $\varphi$ est une matrice de permutation paire. 


\section{Références}

[1] P. M. Cohn, Free rings and their relations, London Math. Society Monographs, Academic Press, London, 1971.

[2] G. Karpilovsky, The Schur multiplier, London Math. Society Monographs, Clarendon Press, Oxford, 1987.

[3] J. Milnor, Introduction to algebraic K-theory, Annals of Mathematics Studies, vol. 72, Princeton University Press and University of Tokyo Press, Princeton, 1971.

[4] I. Porteous, Topological geometry, Cambridge University Press, Cambridge, 2e édition, 1981.

[5] J. ScHuR, Über die Darstellung der symmetrischen und der alternierenden Gruppen durch gebrochene lineare Substitutionen, J. für Reine Angew. Math. 139 (1911), 155-250.

C.K. : Institut de Recherche Mathématique Avancée

Université Louis Pasteur - C.N.R.S.

7 rue René Descartes

67084 Strasbourg Cedex, France

E-mail : kassel@math.u-strasbg.fr

Fax : +33(0)388619069

C.R. : Mathématiques

Université du Québec à Montréal

Montréal, CP 8888, succ. Centre Ville, Canada H3C 3P8

E-mail : reutenauer.christophe@uqam.ca

Fax : +514987 8935

(12 novembre 1996) 